\title{
Investigation of the Mechanism of Shengmai Injection on Sepsis by Network Pharmacology Approaches
}

\author{
Juan Lu, ${ }^{1}$ Xinkai Lyu $\left(\mathbb{D},{ }^{1}\right.$ Ruiping Chai, ${ }^{1}$ Yue Yu, ${ }^{1}$ Minghui Deng, ${ }^{1}$ Xia Zhan, ${ }^{2}$ \\ Zhengqi Dong $\left(\mathbb{D},{ }^{1}\right.$ and Xi Chen $\mathbb{1}^{1}$ \\ ${ }^{1}$ Institute of Medicinal Plant Development, Chinese Academy of Medical Sciences, Peking Union Medical College, \\ Beijing 100094, China \\ ${ }^{2}$ Key Laboratory of Cleaner Production and Integrated Resource Utilization of China National Light Industry, \\ Beijing Technology and Business University, Beijing 100048, China \\ Correspondence should be addressed to Zhengqi Dong; zhengqi_dong@163.com and Xi Chen; chenx_implad@163.com
}

Received 2 March 2020; Accepted 18 June 2020; Published 3 August 2020

Academic Editor: Jeng-Ren Duann

Copyright (c) 2020 Juan Lu et al. This is an open access article distributed under the Creative Commons Attribution License, which permits unrestricted use, distribution, and reproduction in any medium, provided the original work is properly cited.

\begin{abstract}
Shengmai injection (SMI) contains Ginsen Radix et Rhizoma Rubra, Ophiopogon japonicus, and Schisandrae Chinensis Fructus. It is used as a supportive herbal medicine in the management of sepsis, systemic inflammatory response syndrome, and septic or hemorrhagic shock. An UPLC method was established to identify and evaluate SMI fingerprints. Fingerprint similarities of 9 batches of SMI were compared. The network platform, "TCM-components-core targets-key pathways," was established, and the mechanism of SMI in the treatment of sepsis was investigated. The similarity of 9 batches of SMI fingerprints was greater than 0.91 . 44 peaks were selected as the common peaks, of which 11 peaks were identified. KEGG functional pathway analysis showed SMI was mainly involved in the pathways of cancer, cell cycle, and p53 signaling, suggesting SMI protects multiple organs via regulating immunity, inflammation, apoptosis, and energy metabolism. GO enrichment analysis showed active SMI components regulated various biological processes and altered the pathophysiology of sepsis. The interplays between SMI and multiple energy metabolism signaling cascades confer protection from life-threatening multiple organ failure in sepsis.
\end{abstract}

\section{Introduction}

Sepsis is a deregulated body response to infection, triggering inflammatory reactions that can cause systemic symptoms and damage multiple organs. Release of cytokines mediates uncontrolled inflammatory cascades that result in dysfunction and failure of multiple major organs and septic shock $[1,2]$. Managing infection is the most critical strategy for sepsis therapy. However, these treatments could cause various side effects [3]. Clinically, sepsis is managed with early use of antibiotics and glucocorticoids. Traditional Chinese medicine, including Xuebijing injection [2, 4], Shenfu injection [5], and SMI [6], provides supportive effects in sepsis treatment.

Shengmai injection (SMI) origins from the ancient prescription of Chinese medicine Shengmaiyin; it contains Ginsen Radix et Rhizoma Rubra, Ophiopogon japonicus, and
Schisandrae Chinensis Fructus. It invigorates Qi, nourishes Yin, and promotes blood circulation. SMI is used as add-on for supportive treatment in managing patients with sepsis, systemic inflammatory syndrome, and septic or hemorrhagic shock [7-9]. The frequency of adverse events associated with SMI is low $[10,11]$. There have been few reports on the evaluation of SMI effective components and their underlying mechanisms despite they are used as supportive interventions for sepsis treatment. It is a compound with multiple components targeting multiple molecular networks; exploring its complex antisepsis mechanism in a suitable model is of great importance for sepsis treatment [12]. Network pharmacology is a useful tool for systemic investigation of the mechanisms of multiple component drugs $[13,14]$. Its approach has been used to study "compound-protein/gene-disease" pathways which reveal complexities among drugs, biological systems, and diseases from 
a network perspective. Network pharmacology provides insights into the complex interrelationships between the active ingredients of traditional Chinese compounds and molecular mechanisms [15].

We established a fingerprint method to detect and represent chemical information of SMI. We mapped potential targets of SMI bioactive ingredients that may regulate the progress of sepsis using a network pharmacology approach. Our findings shed light on further understanding of the mechanisms of SMI in treating complex diseases such as sepsis.

\section{Materials and Methods}

2.1. Equipment and Reagents. Water Acquity H-class UPLC, equipped with quaternion high-pressure pump, automatic sampler, and PDA detector (Milford, USA); METTLER AB265-S electronic analytical balance (Zurich, Switzerland); and SB25-12DT ultrasonic cleaner (Ningbo, China) were used. Standard ginsenoside Rb1 (no. 171018), ginsenoside Rb2 (no. 171009), ginsenoside Rd (no. 170530), ginsenoside $\operatorname{Re}$ (no. 170924), ginsenoside RF (no. 171126), ginsenoside Rg1 (no. 180105), Ophiopogon D (no. 171126), schisandrol A (no. 180109), and schisandrin A (no. 171231) were all purchased from Shanghai Ronghe Medical Pharmaceutical Technology Co., Ltd (Shanghai, China). Ginsenoside Rb3 (no. 111686-201504) and ginsenoside Rg2 (no. 111779200801) were purchased from the National Institutes for Food and Drug Control (Beijing, China). The purity of all of the above standards was above $98.0 \%$. Acetonitrile and methanol were purchased from Merck (chromatographically pure, Darmstadt, Germany). Distilled water was purchased from Watson (Hong Kong, China). There were 9 batches of SMI: S1, 16120401005; S2, 160502; S3, 17071014; S4, 17040423; S5, 1704252; S6, 17091302; S7, 17092903; S8, 17061103; and S9, 17053005.

\subsection{Standards and Sample Solution Preparation.} Standard stock solutions of ginsenoside Rb1 (9.49 mg), ginsenoside Rb2 (10.22 mg), ginsenoside Rb3 (6.69 mg), ginsenoside $\mathrm{Rd}$ (6.24 mg), ginsenoside Re (6.24 mg), ginsenoside Rf (7.35 mg), ginsenoside Rg1 (13.24 mg), ginsenoside Rg2 (6.58 mg), Ophiopogon D (5.12 mg), schisandrol A (5.60 mg), and schisantherin A (3.27 mg) were dissolved in $5 \mathrm{ml}$ methanol followed by sonication, respectively. Mixture of standard solution was filtered through the $0.22 \mu \mathrm{m}$ membrane in a $5 \mathrm{~mL}$ volumetric flask. Final concentration of each standard in the mixture was 37.96, 40.88, 26.76, 24.96, $24.96,29.40,52.96,26.32,20.48,17.92$, and $10.46 \mu \mathrm{g} \cdot \mathrm{mL}^{-1}$, respectively. SMI solution of each batch was filtered through the $0.22 \mu \mathrm{m}$ membrane before analysis.

2.3. UPLC Conditions. The analyte was separated by a Waters Acquity UPLC BEH C18 $(2.1 \mathrm{~mm} \times 50 \mathrm{~mm}, 1.7 \mu \mathrm{m})$ column. The mobile phases used were solvent $A$ (acetonitrile) and solvent $B$ (water) with gradient elution (Table 1). The analysis was carried out at a flow rate of $0.3 \mathrm{~mL} / \mathrm{min}$. The column temperature was set to $40^{\circ} \mathrm{C}$. UV detection
TABLE 1: Mobile phase and proportion of qualitative and quantitative chromatographic conditions.

\begin{tabular}{lcc}
\hline Time $(\mathrm{min})$ & $A(\%)$ & $B(\%)$ \\
\hline 0 & 19 & 81 \\
3 & 19 & 81 \\
12 & 26.8 & 73.2 \\
15 & 32 & 68 \\
23 & 32 & 68 \\
23.1 & 44 & 56 \\
35 & 66.8 & 33.2 \\
\hline
\end{tabular}

wavelength was over the range of 190 to $400 \mathrm{~nm}$. $5 \mu \mathrm{L}$ of the sample was injected. $210 \mathrm{~nm}$ was selected as the extraction wavelength of the fingerprints.

2.4. Precision of the Method. Method precision was determined by analyzing the same sample SMI (S1, 16120101005) five consecutive times in a day. The peak of schisantherin A was used as the reference peak. Relative standard deviation (RSD) was calculated from the relative peak area (RPA) or relative retention time (RRT) of each characteristic peak.

2.5. Sample Stability. Sample stability was evaluated using the same SMI (S1, 16120401005) after 0, 2, 4, 6, 8, 12, and 24 hours. The peak of schisantherin A was used as the reference peak. RSD was calculated from RPA or RRT of each peak to the reference peak from the chromatographic profiles of samples.

2.6. Repeatability. Repeatability was evaluated by analyzing six independently prepared SMI samples. The peak of schisantherin A was used as the reference peak. RSD was calculated from RPA or RRT of each peak to the reference peak from the chromatographic profiles of samples.

2.7. Information about Databases and Software of Network Pharmacology. TCMSP database (https://tcmspw.com/ tcmsp.php), PubChem CID (https://pubchem.ncbi.nlm. nih.gov/search/), STITCH (http://stitch.embl.de/), Human Phenotype Ontology (HPO, https:/hpo.jax.org/app/), STRING database (https://string-db.org), OMIM database (https://omim.org/), and DAVID database (https://david. ncifcrf.gov/summary.jsp) were used. Cytoscape software v3.5.1 was used.

2.8. Network Construction. Data acquisition and processing were done in databases which include SciFinder and TCMSP. Additionally, PubChem CID for each active ingredient of SMI was obtained from PubChem. We used SMILES format in STITCH chemical association networks and obtained the interaction complex between SMI bioactive components and the potential target protein in humans. Using HPO as a tool, we annotated and analyzed the core protein targets that participate in sepsis. The primary as well as predicted interactions between SMI target proteins and proteins involved in sepsis were analyzed in the STRING 


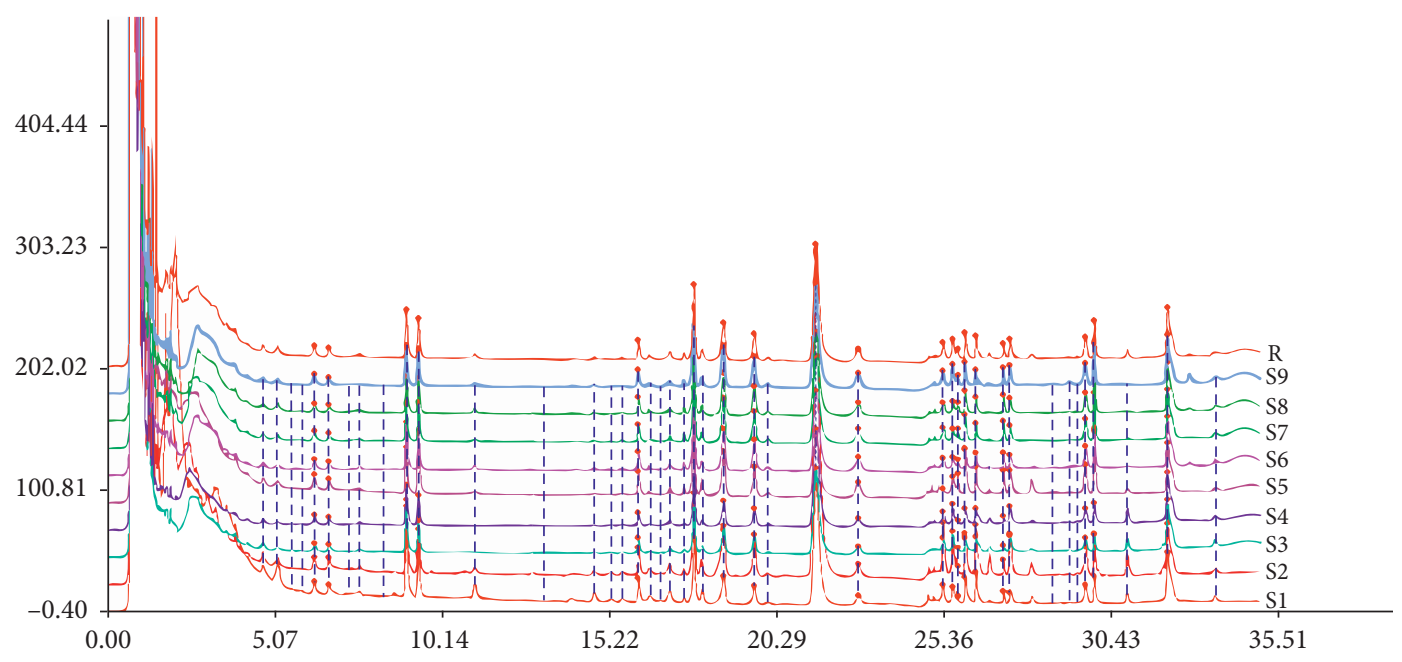

FIgURE 1: UPLC fingerprints of 9 batches of SMI.
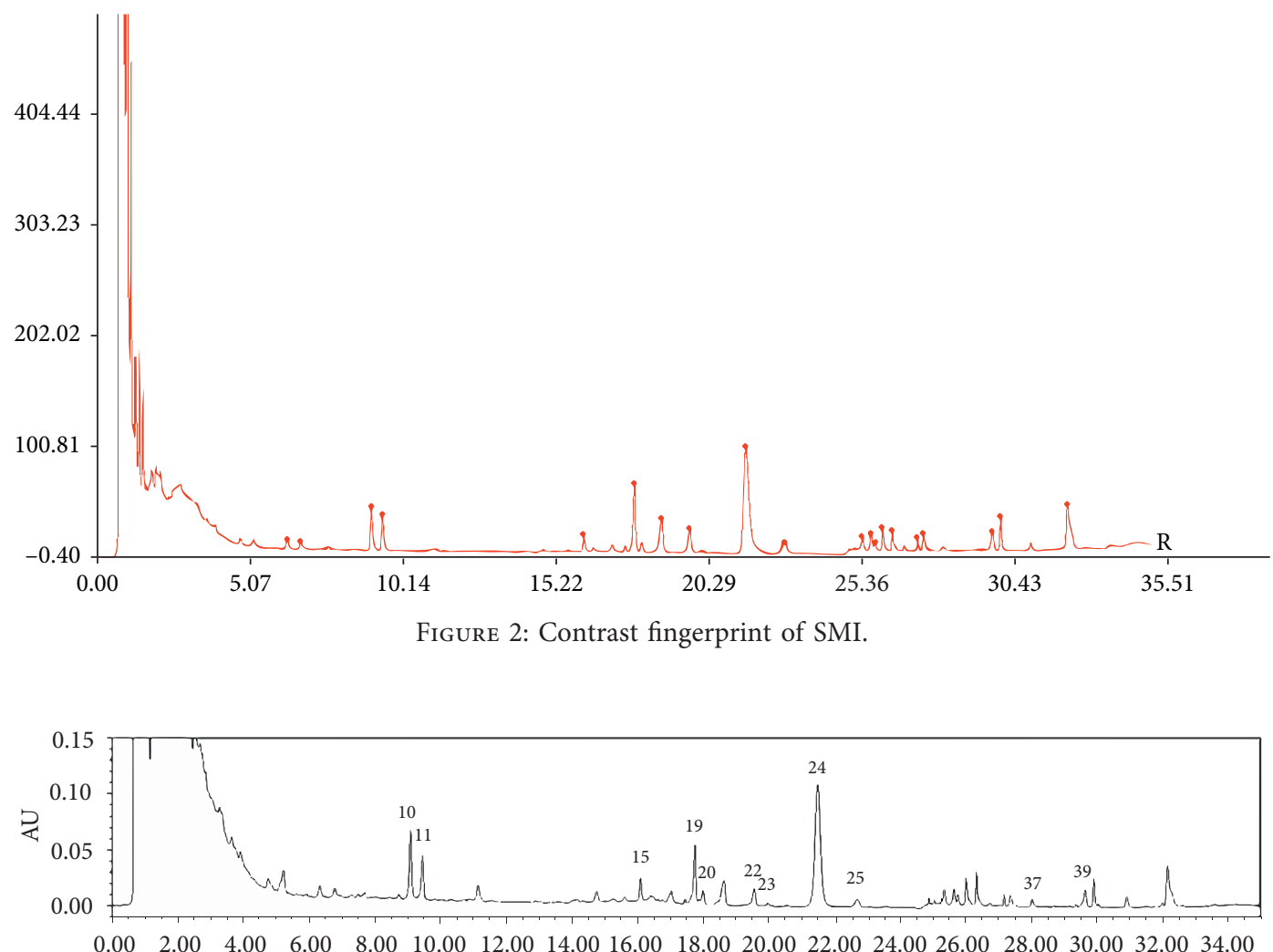

(a)

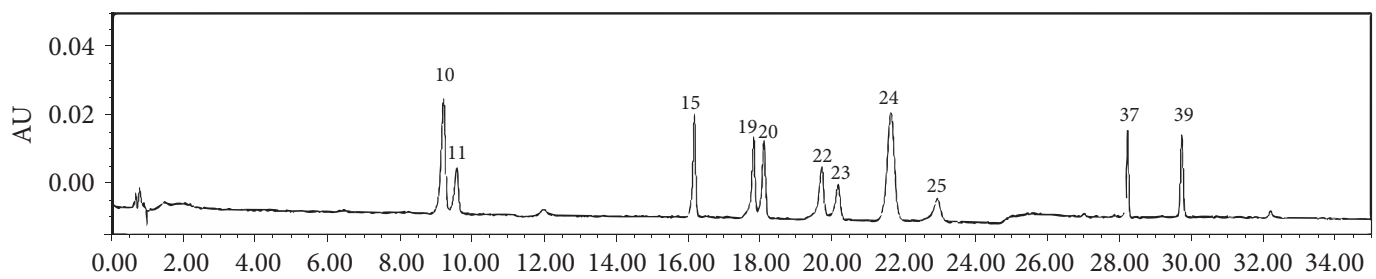

(b)

FIGURE 3: UPLC chromatogram of SMI and mixed reference at $210 \mathrm{~nm}$ : (a) samples of SMI (S1); (b) mixed reference (10, ginsenoside Rg1; 11, ginsenoside Re; 15, ginsenoside Rf; 19, ginsenoside Rb1; 20, ginsenoside Rg2; 22, ginsenoside Rb2; 23, ginsenoside Rb3; 24, schisandrol A; 25, ginsenoside Rd; 37, Ophiopogon D; and 39, schisandrin A). 
TABLE 2: Comparison of similarity of Shengmai injection in different batches.

\begin{tabular}{lcccccccccc}
\hline & S1 & S2 & S3 & S4 & S5 & S6 & S7 & S8 & S9 & Control fingerprints \\
\hline S1 & 1 & 0.954 & 0.983 & 0.976 & 0.916 & 0.938 & 0.944 & 0.942 & 0.953 & 0.968 \\
S2 & 0.954 & 1 & 0.943 & 0.945 & 0.821 & 0.869 & 0.89 & 0.885 & 0.922 & 0.914 \\
S3 & 0.983 & 0.943 & 1 & 0.992 & 0.931 & 0.962 & 0.967 & 0.965 & 0.973 & 0.986 \\
S4 & 0.976 & 0.945 & 0.992 & 1 & 0.927 & 0.945 & 0.952 & 0.95 & 0.967 & 0.978 \\
S5 & 0.916 & 0.821 & 0.931 & 0.927 & 1 & 0.944 & 0.944 & 0.939 & 0.931 & 0.963 \\
S6 & 0.938 & 0.869 & 0.962 & 0.945 & 0.944 & 1 & 0.996 & 0.996 & 0.983 & 0.98 \\
S7 & 0.944 & 0.89 & 0.967 & 0.952 & 0.944 & 0.996 & 1 & 0.998 & 0.989 & 0.984 \\
S8 & 0.942 & 0.885 & 0.965 & 0.95 & 0.939 & 0.996 & 0.998 & 1 & 0.989 & 0.98 \\
S9 & 0.953 & 0.922 & 0.973 & 0.967 & 0.931 & 0.983 & 0.989 & 0.989 & 1 & 0.98 \\
Control & 0.968 & 0.914 & 0.986 & 0.978 & 0.963 & 0.98 & 0.984 & 0.98 & 0.98 & 1 \\
fingerprints & & & & & & & & & & \\
\hline
\end{tabular}

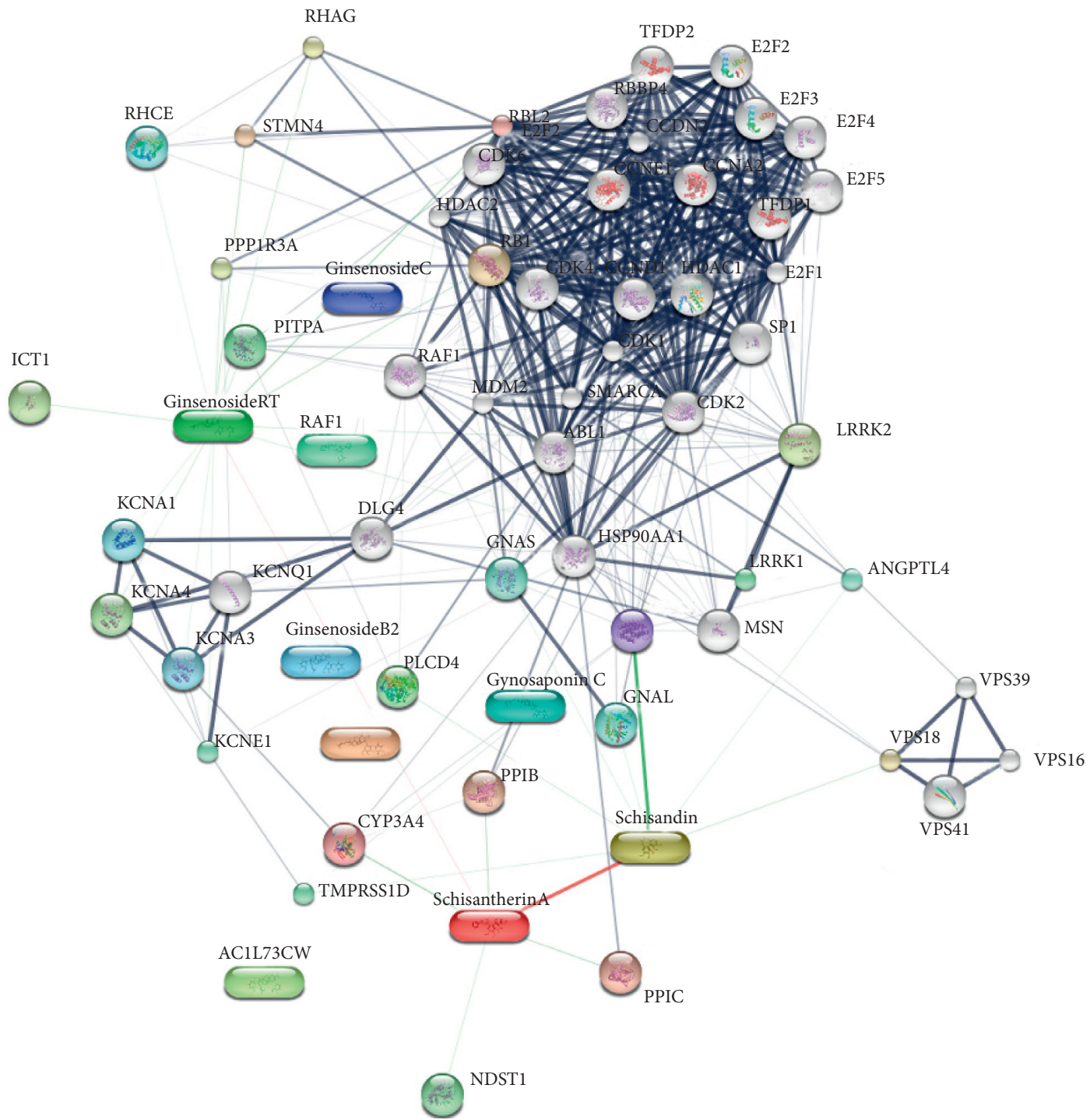

FIGURE 4: Prediction of component-target of Shengmai injection by STITCH.

database. We collected core proteins that are highly associated with sepsis, while proteins with low correlation were filtered out [16]. The molecular interplays between SMI key targets and sepsis proteins were visualized in the Cytoscape platform. We calculated the degree, betweenness, and closeness of the targets; proteins with topological parameters greater than the corresponding median values were considered as major hits. The selected proteins were validated in 
TABle 3: Prediction of pharmacodynamic targets of Shengmai injection.

\begin{tabular}{|c|c|}
\hline No. & Targets \\
\hline 1 & E2F5 \\
\hline 2 & HDAC2 \\
\hline 3 & VPS33A \\
\hline 4 & TFDP2 \\
\hline 5 & MSN \\
\hline 6 & KCNE1 \\
\hline 7 & $\mathrm{RB} 1$ \\
\hline 8 & CDK1 \\
\hline 9 & TFDP1 \\
\hline 10 & MDM2 \\
\hline 11 & VPS16 \\
\hline 12 & VPS39 \\
\hline 13 & $\mathrm{E} 2 \mathrm{~F} 4$ \\
\hline 14 & E2F2 \\
\hline 15 & CDK2 \\
\hline 16 & VPS41 \\
\hline 17 & KCNA4 \\
\hline 18 & HSP90AA1 \\
\hline 19 & CDK4 \\
\hline 20 & CDK6 \\
\hline 21 & HDAC1 \\
\hline 22 & CCNA2 \\
\hline 23 & E2F1 \\
\hline 24 & RBBP4 \\
\hline 25 & ABL1 \\
\hline 26 & SP1 \\
\hline 27 & CCND2 \\
\hline 28 & E2F3 \\
\hline 29 & RBL2 \\
\hline 30 & CCNE1 \\
\hline 31 & KCNA3 \\
\hline 32 & KCNA1 \\
\hline 33 & RAF1 \\
\hline 34 & GNAS \\
\hline 35 & LRRK1 \\
\hline 36 & STMN4 \\
\hline 37 & HMOX1 \\
\hline 38 & PPP1R3A \\
\hline 39 & RHAG \\
\hline 40 & PTGES3 \\
\hline 41 & GNAL \\
\hline 42 & CCND1 \\
\hline 43 & CYP3A4 \\
\hline 44 & PPIB \\
\hline 45 & LRRK2 \\
\hline 46 & GALE \\
\hline 47 & PPIC \\
\hline 48 & RHCE \\
\hline 49 & VPS18 \\
\hline 50 & ANGPTL4 \\
\hline 51 & ICT1 \\
\hline 52 & DLG4 \\
\hline 53 & CKS2 \\
\hline 54 & TMPRSS11D \\
\hline 55 & KCNQ1 \\
\hline 56 & CCNB1 \\
\hline 57 & CCNB2 \\
\hline 58 & CCND3 \\
\hline 59 & CDC20 \\
\hline
\end{tabular}

TABle 3: Continued.

\begin{tabular}{lc}
\hline No. & Targets \\
\hline 60 & CDC37 \\
61 & CDC6 \\
62 & CDKN1B \\
\hline
\end{tabular}

the OMIM database to establish protein-disease association and construct "SMI Targets-Sepsis Targets" network.

2.9. Prediction of the SMI-Antisepsis Mechanism. A list of the selected top 20 key proteins was uploaded to the DAVID database for functional annotation and enrichment analysis to obtain the main pathways and network distribution that confer potential mechanisms for SMI treatment. Only pathways with $p<0.05$ were considered for mechanism prediction.

\section{Results and Discussion}

3.1. Establishment of SMI Fingerprints and the Results of Methodological Evaluation. The RSDs of RPA and RRT for precision, repeatability, and sample stability were lower than $3 \%$, respectively. The results showed that the fingerprint method developed for analysis of SMI is reliable and applicable. Figures 1 and 2 show the UPLC chromatogram fingerprints of 9 batches of SMI.

3.2. Reference Peak and Common Peak. The peak of schisantherin A was used as the reference peak; it showed as an intense peak with preferable chromatographic peak resolution and RRT. Peaks that existed in all SMI samples were appointed as "common peaks." 44 common peaks were detected in SMI samples, in which 11 peaks were identified (Figure 3): ginsenoside Rg1 (peak \#10), ginsenoside Re (peak \#11), ginsenoside Rf (peak \#15), ginsenoside Rb1(peak \#19), ginsenoside Rb2 (peak \#20), ginsenoside Rb2 (peak \#22), ginsenoside Rb3 (peak \#23), schisandrol A (peak \#24), ginsenoside Rd (peak \#25), Ophiopogonin D (peak \#37), and schisantherin A (peak \#39).

3.3. Similarity of Fingerprints of 9 Batches of SMI. The similarities of all chromatographic patterns among the samples (Table 2) were calculated using software "Chromatographic Fingerprints of Traditional Chinese Medicine, version: 2004A." The similarities of 9 SMI batches were greater than 0.91. Therefore, our method was precise, stable, reproducible, and reliable.

3.4. Putative Targets of SMI Ingredients. The SMI components were screened by TCMSP, and the criteria are OB (oral bioavailability) $\geq 30 \%$ and, meanwhile, DL (drug-like) $\geq 0.18$ [17-19]. All the components were confirmed in the PubChem database [19] (total 9 bioactive SMI components: ginsenoside $\mathrm{Rb} 1$, ginsenoside $\mathrm{Rb} 2$, ginsenoside $\mathrm{Re}$, 
TABLE 4: Prediction of sepsis closely related proteins in the human body.

\begin{tabular}{|c|c|}
\hline No. & Targets \\
\hline 1 & CYBB \\
\hline 2 & RMRP \\
\hline 3 & RAG1 \\
\hline 4 & RAG2 \\
\hline 5 & TGM1 \\
\hline 6 & LIG4 \\
\hline 7 & SEMA3D \\
\hline 8 & NIPAL4 \\
\hline 9 & SEMA3C \\
\hline 10 & TCF3 \\
\hline 11 & MYH11 \\
\hline 12 & ATP7A \\
\hline 13 & WAS \\
\hline 14 & WIPF1 \\
\hline 15 & GALT \\
\hline 16 & HLA-B \\
\hline 17 & G6PC3 \\
\hline 18 & DCLRE1C \\
\hline 19 & NRTN \\
\hline 20 & ABCA 12 \\
\hline 21 & PIK3R1 \\
\hline 22 & IGHM \\
\hline 23 & NFKB2 \\
\hline 24 & BTK \\
\hline 25 & NCF1 \\
\hline 26 & BLNK \\
\hline 27 & $\mathrm{APC}$ \\
\hline 28 & LRRC8A \\
\hline 29 & ELANE \\
\hline 30 & ACTG2 \\
\hline 31 & CYP4F22 \\
\hline 32 & $\mathrm{AK} 2$ \\
\hline 33 & CD79A \\
\hline 34 & CD79B \\
\hline 35 & NCF2 \\
\hline 36 & IKZF1 \\
\hline 37 & ALOXE3 \\
\hline 38 & NCF4 \\
\hline 39 & SERAC1 \\
\hline 40 & LIPN \\
\hline 41 & CHD7 \\
\hline 42 & IGLL1 \\
\hline 43 & RET \\
\hline 44 & PLEC \\
\hline 45 & CTNNB1 \\
\hline 46 & ECE1 \\
\hline 47 & $\mathrm{ADA}$ \\
\hline 48 & IL2RG \\
\hline 49 & ITGB4 \\
\hline 50 & GDNF \\
\hline 51 & MUT \\
\hline 52 & ALOX12B \\
\hline 53 & EDN3 \\
\hline 54 & EDNRB \\
\hline 55 & IL7R \\
\hline 56 & FERMT3 \\
\hline 57 & TFRC \\
\hline 58 & CYBA \\
\hline
\end{tabular}

ginsenoside Rf, ginsenoside Rg1, ginsenoside Rg2, schisandrol A, schisantherin A, and Ophiopogon D. Their chemical structures and molecular properties were analyzed and uploaded to the STITCH database for predicting targets that interact with SMI ingredients [20]. A total of 62 targets (Figure 4 and Table 3 ) showed potential interaction with 9 SMI ingredients.

3.5. Acquisition of Known Therapeutic Sepsis Targets. Sepsis targets were collected from the HPO database. The keyword "sepsis" was used to search known therapeutic targets for sepsis in humans [21]. A total of 58 sepsis targets (Table 4) were acquired from the HPO database, and targets were further verified in the NCBI database.

3.6. Results of Network Construction. The putative targets of SMI active ingredients and sepsis disease targets were determined based on the protein-protein interactions [22]. The interplays amongst SMI targets, known therapeutic targets for sepsis, and interactional human targets were combined to construct the network. The network illustrates the relationship between SMI targets and sepsis targets. The overall interaction network (Figure 5) was visualized using Cytoscape (sepsis targets in red circles and SMI targets in blue squares); the larger a node, the more targets it contains and more important in sepsis management. Targets with higher values of "degree," "betweenness," "closeness," and "coreness" (above the median value of all the network nodes) were identified $[23,24]$. Targets which might play unimportant roles in the network according to the topological features were discarded [25]. Median value of "degree," "betweenness," and "closeness" was 19,0.014, and 0.449, respectively. Top twenty proteins were selected as key sepsis therapeutic targets (Figure 6 and Table 5), including ABL1, CCND1, CDK family (CDK1, CDK2, CDK6, and CDKN1B), RB1, HSP90AA1, SMARCA4, RBL2, CTNNB1, MDM2, SP1, LRRK1, BTK, PIK3R1, TMPRSS11D, ACTG2, CD79A, and RET.

Sepsis causes life-threatening organ dysfunction due to a host's complex systemic inflammatory response to infection [26]. In the present study, we identified core proteins that may play important roles in SMI-supportive treatment in sepsis. ABL1 is a tyrosine-protein kinase which is important for cell growth and survival, cytoskeleton remodeling in response to extracellular stimuli, autophagy, and apoptosis [27-29]. It also regulates multiple pathological signaling cascades during infection that alter vascular permeability and the endothelium barrier in inflammation [30].

Cyclin-dependent kinase 1 (CDK1) is a member of the Ser/Thr protein kinase family. Its kinase activity is controlled by cyclin $[31,32]$. CCND1/CDK4 and CDK2 are critical for $\mathrm{G} 1 / \mathrm{S}$ phase transition. It has been shown rat kidney injury is associated with G1 phase arrest in cecal ligation and puncture- (CLP-) induced sepsis, while upregulation of CCND1/CDK4 and CCNE/CDK2 activates $\mathrm{Rb}$ leading to revival of cell cycle progress and recovery of kidney function 


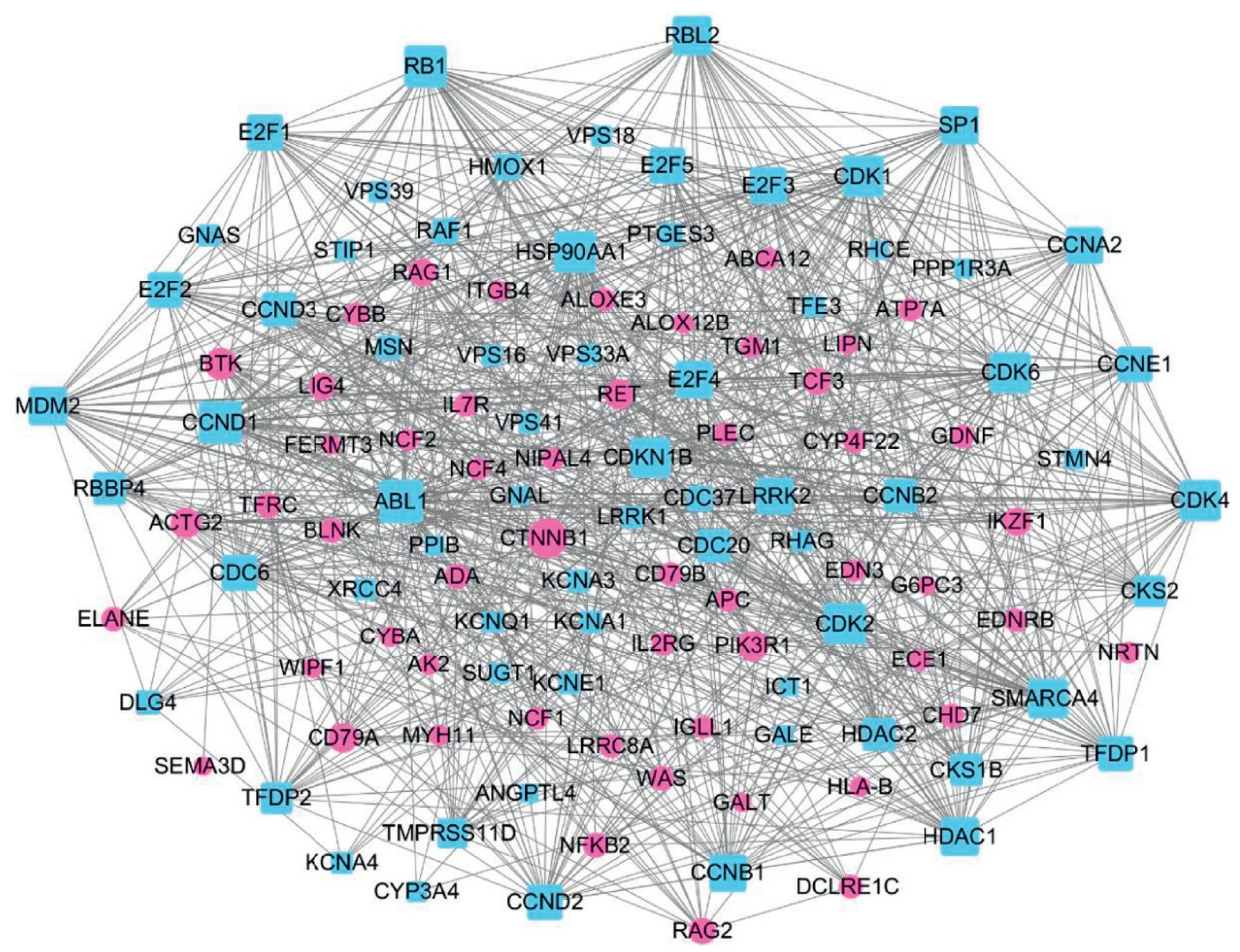

FIGURE 5: Prediction of protein interaction between SMI and sepsis by STRING (blue-square nodes are targets of SMI; red circular nodes are targets of sepsis).

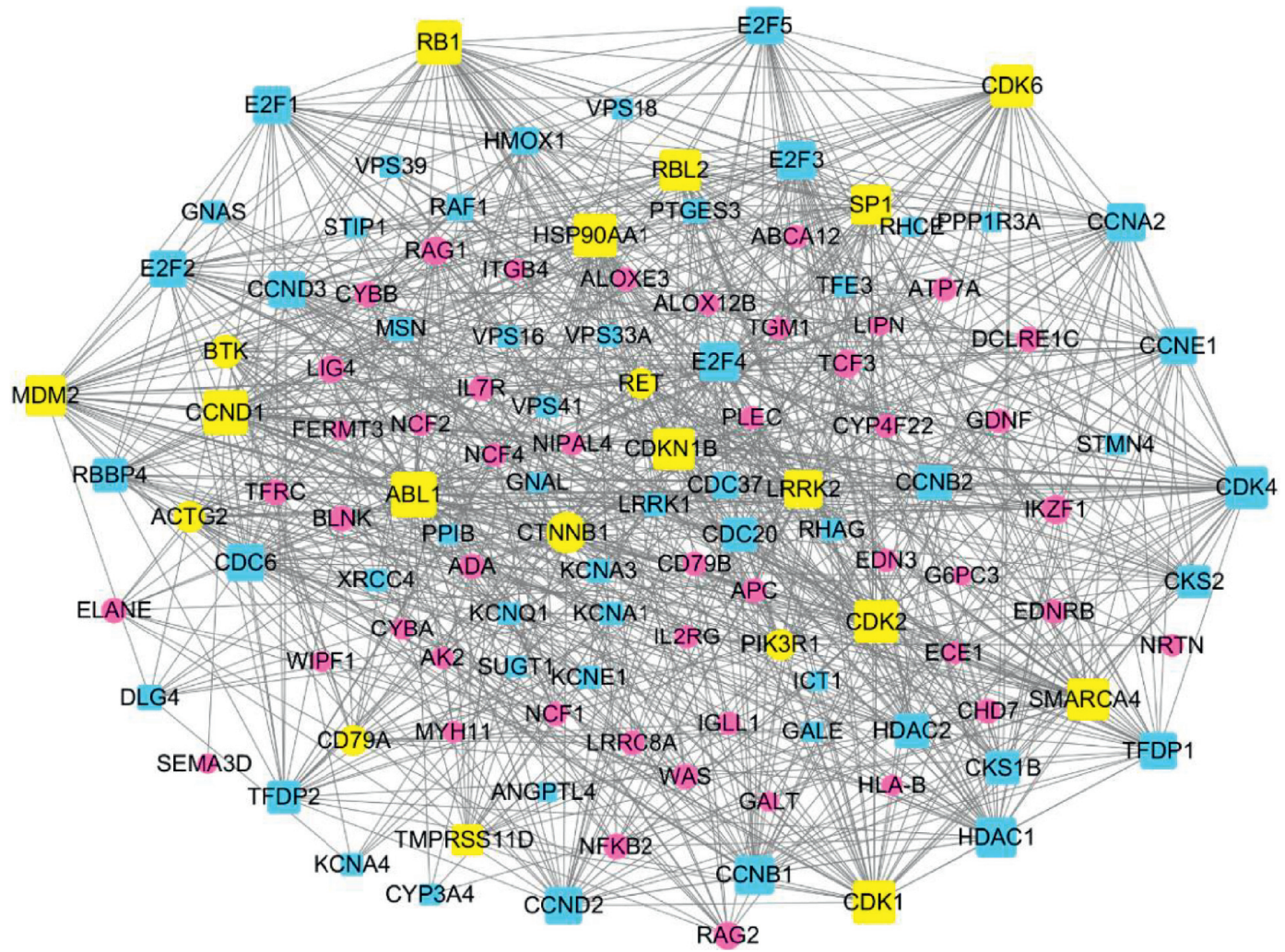

Figure 6: Key targets of the SMI-sepsis protein interaction network (yellow is the key target; blue-square nodes are SMI targets; and red circular nodes are sepsis targets). 
TABLE 5: Key targets and topological parameters of the network on SMI in the treatment of sepsis.

\begin{tabular}{|c|c|c|c|c|c|}
\hline No. & Gene name & Protein name & Degree & $\begin{array}{l}\text { Closeness } \\
\text { centrality }\end{array}$ & $\begin{array}{c}\text { Betweenness } \\
\text { centrality }\end{array}$ \\
\hline 1 & ABL1 & Tyrosine-protein kinase ABL1 & 43 & 0.548 & 0.101 \\
\hline 2 & CCND1 & G1/S-specific cyclin-D1 & 42 & 0.522 & 0.046 \\
\hline 3 & RB1 & Retinoblastoma-associated protein & 41 & 0.509 & 0.037 \\
\hline 4 & $\mathrm{CDK} 2$ & Cyclin-dependent kinase 2 & 41 & 0.52 & 0.026 \\
\hline 5 & HSP90AA1 & Heat-shock protein HSP 90 -alpha & 40 & 0.531 & 0.077 \\
\hline 6 & CDK1 & Cyclin-dependent kinase 1 & 39 & 0.509 & 0.023 \\
\hline 7 & CDK6 & Cyclin-dependent kinase 6 & 39 & 0.502 & 0.015 \\
\hline 8 & SMARCA4 & Transcription activator BRG1 & 37 & 0.511 & 0.037 \\
\hline 9 & CDKN1B & Cyclin-dependent kinase inhibitor $1 \mathrm{~B}$ & 37 & 0.506 & 0.031 \\
\hline 10 & RBL2 & Retinoblastoma-like protein 2 & 36 & 0.482 & 0.04 \\
\hline 11 & CTNNB1 & Catenin beta- 1 & 36 & 0.509 & 0.036 \\
\hline 12 & MDM2 & E3 ubiquitin-protein ligase $\mathrm{Mdm} 2$ & 35 & 0.488 & 0.025 \\
\hline 13 & SP1 & Transcription factor $\mathrm{Sp} 1$ & 34 & 0.504 & 0.027 \\
\hline 14 & LRRK1 & Leucine-rich repeat serine/threonine-protein kinase 1 & 31 & 0.515 & 0.115 \\
\hline 15 & BTK & Tyrosine-protein kinase BTK & 23 & 0.486 & 0.038 \\
\hline 16 & PIK3R1 & Phosphatidylinositol 3-kinase regulatory subunit alpha & 21 & 0.47 & 0.054 \\
\hline 17 & TMPRSS11D & Transmembrane protease serine 11D & 20 & 0.463 & 0.041 \\
\hline 18 & ACTG2 & Actin, gamma-enteric smooth muscle & 20 & 0.454 & 0.025 \\
\hline 19 & CD79A & B-cell antigen receptor complex-associated protein alpha chain & 19 & 0.458 & 0.058 \\
\hline 20 & RET & Proto-oncogene tyrosine-protein kinase receptor Ret & 19 & 0.463 & 0.032 \\
\hline
\end{tabular}

48 hours after CLP $[33,34]$. The findings demonstrate that cell cycle arrest occurs in sepsis, and drugs that regulate cell cycle proteins may be a means to rescue organ injury [35]. In addition, the targets of SMI are more involved in DNA replication and transcription; for example, MDM2, E3 ubiquitin ligase, mediates ubiquitination and degradation of p53. It mediates apoptosis in organ injury and malignant transformation [36]. SMI may inhibit MDM2 and keep p53 active; therefore, it promotes cells staying in the G1/G2 phase and alleviates cell injury in sepsis.

Molecular chaperone heat-shock protein (HSP 90) is extensively expressed by cells, and its expression increases upon stimulation [37]. HSPs are associated with multiple organ failure in sepsis [38]. In the vast immune response in sepsis, stressed cells release HSPs that are regarded as "danger signal" to neighboring and immune cells [39]. HSP90- $\alpha$ has been shown to interact with about 200 client proteins, including signal proteins in the inflammatory pathway such as NF- $\kappa \mathrm{B}$, Akt, and IKK, to interfere inflammation [40-42]. Moreover, HSP90- $\alpha$, as abundant "chaperone," is one of the main mediators that activates bacteria lipopolysaccharide. It interacts with proteins in the $\mathrm{PI} 3 \mathrm{~K} / \mathrm{Akt}$ pathway and is essential in promoting the immune response and improving host defense to pathogens. Inhibition of HSP90- $\alpha$ prevents severe sepsis-associated acute lung injury; therefore, block HSP90- $\alpha$ offers a novel treatment for lung injury in sepsis [43-45].

BTK (tyrosine-protein kinase) is a component of the tolllike receptor (TLR) pathway and plays important roles in innate and adaptive immunity. Key target CD79A is required for efficient differentiation of pro- and pre-B-cells. It cooperates with $\mathrm{CD} 79 \mathrm{~B}$ and bounds to the B-cell antigen receptor complex (BCR) for initiation of the signal transduction cascade. It is pivotal in regulating immunity and inflammation [46]. Network pharmacology revealed SMI
TABLE 6: Analysis of major metabolic pathways related to sepsis in KEGG.

\begin{tabular}{lccc}
\hline No. & Name & Count & $p$ value \\
\hline 1 & Pathways in cancer & 11 & $6.2 \times 10^{-9}$ \\
2 & Cell cycle & 9 & $1.2 \times 10^{-9}$ \\
3 & p53 signaling pathway & 5 & $4.7 \times 10^{-5}$ \\
4 & Melanoma & 5 & $5.6 \times 10^{-5}$ \\
5 & Non-small cell lung cancer & 4 & $5.8 \times 10^{-4}$ \\
6 & B-cell receptor signaling pathway & 3 & $2.3 \times 10^{-2}$ \\
7 & Colorectal cancer & 3 & $2.8 \times 10^{-2}$ \\
8 & ErbB signaling pathway & 3 & $3 \times 10^{-2}$ \\
\hline
\end{tabular}

represses BTK expression/activation, blocks signals through multiple pathways (TLR, B-cell antigen receptor signal, and apoptosis), and consequently ameliorates cell apoptosis and organ injury. SMI acts as a whole, and each formula has its corresponding targets/syndromes; thus, SMI prescription acts on multiple key targets, and the network pharmacology study of SMI provides insights into understanding its fundamental mechanisms.

3.7. KEGG and GO Analysis. To cluster the biological functions of SMI and its targets, data were uploaded to KEGG, and results revealed SMI active formulae target pathways including cancer, cell cycle, p53, B-cell receptor, and ErbB pathways (Table 6). SMI regulates the interplay and synergy among the pathways of immunity, inflammation, and apoptosis to protect cellular and organ injury in sepsis. p53 pathway regulates mitochondrial fission and mitochondrial biogenesis via AMPK, and it alters PKM2dependent glycolysis. Global deletion of PKM2 results in systemic inflammation in mice [47]. Our GO analysis (Table 7) showed that SMI ingredients regulate multiple 
TABLe 7: GO biological analysis.

\begin{tabular}{|c|c|c|c|}
\hline No. & Name & Count & $p$ value \\
\hline 1 & Cell cycle & 10 & $3.5 \times 10^{-7}$ \\
\hline 2 & Phosphorylation & 9 & $6.1 \times 10^{-6}$ \\
\hline 3 & Phosphorus metabolic process & 9 & $2.6 \times 10^{-5}$ \\
\hline 4 & Intracellular signaling cascade & 9 & $1.6 \times 10^{-4}$ \\
\hline 5 & Regulation of transcription & 9 & $1.8 \times 10^{-2}$ \\
\hline 6 & Mitotic cell cycle & 8 & $4.1 \times 10^{-7}$ \\
\hline 7 & Protein amino acid phosphorylation & 8 & $2.1 \times 10^{-5}$ \\
\hline 8 & Positive regluation of macromolecule metabolic process & 8 & $1.0 \times 10^{-4}$ \\
\hline 9 & Interphase & 7 & $5.0 \times 10^{-9}$ \\
\hline 10 & Regulation of cell proliferation & 7 & $5.4 \times 10^{-4}$ \\
\hline 11 & Regulation of transcription, DNA-dependent & 7 & $3.0 \times 10^{-2}$ \\
\hline 12 & Regulation of RNA metabolic process & 7 & $3.3 \times 10^{-2}$ \\
\hline 13 & Regulation of mitotic cell cycle & 6 & $2.6 \times 10^{-15}$ \\
\hline 14 & Hemopoiesis & 6 & $1.5 \times 10^{-5}$ \\
\hline 15 & Hemopoietic or lyphoid organ development & 6 & $2.4 \times 10^{-5}$ \\
\hline 16 & Immune system development & 6 & $3.1 \times 10^{-5}$ \\
\hline 17 & Positive regluation of nitrogen compound metabolic process & 6 & $1.6 \times 10^{-3}$ \\
\hline 18 & Positive regluation of celluar biosynthetic process & 6 & $2.1 \times 10^{-3}$ \\
\hline 19 & Positive regluation of biosynthetic process & 6 & $2.2 \times 10^{-3}$ \\
\hline 20 & Regulation of transcription from RNA polymerase II promoter & 6 & $2.7 \times 10^{-3}$ \\
\hline
\end{tabular}

biological processes including cell cycle, energy metabolism, cellular signal transduction, transcription regulation, and immunity development. Our data indicate the putative role of SMI in alleviating systemic inflammation and deregulating immunity in the host; moreover, it regulates energy utilization and promotes energy homeostasis and therefore ameliorates multiple organ failure associated with sepsis. It is also in agreement with the idea of SMI used in the early phase of sepsis.

\section{Conclusion}

An UPLC method was developed for analysis of SMI fingerprints. Forty-four peaks were selected as common peaks, of which 11 peaks were identified. The consistency in the chromatograms of 9-batch samples reflects the presence of similar chemical constituents (similarities greater than 0.91). The technique was proven to be useful in SMI quality control. A total of 9 active components of SMI target 20 key proteins including ABL1, CDK, HSP90, BTK, PIK3R1, and CD79A. These proteins are enriched in cell cycle, p53 signaling pathway, B-cell receptor signaling pathway, and ErbB pathway. It is likely that the pharmacological mechanisms of SMI in sepsis treatment are of multiple dimensions that are associated with regulation of cell cycle, energy metabolism, cellular signal transduction, transcription regulation, and immunity development. Further experiments are needed to validate our prediction.

\section{Data Availability}

The data used to support the findings of this study are available from the corresponding author upon request.

\section{Conflicts of Interest}

The authors declare no conflicts of interest.

\section{Authors' Contributions}

Juan Lu and Xinkai Lyu contributed equally to this work. $\mathrm{XC}, \mathrm{ZD}$, and JL conceived, designed, and supervised the project. RC, YY, XL, MD, and XZ performed the experiments. JL and XL integrated all the data and wrote the paper.

\section{Acknowledgments}

This research was funded by the National Natural Science Foundation of China (no. 81673667), the CAMS Innovation Fund for Medical Science (CIFMS) (nos. 2016-I2M-3-015 and 2017-I2M-B\&R-09), Beijing Natural Science Committee-Beijing Education Committee Joint Foundation (no. KZ201910011012), and Science and Technology Projects in Liaoning Province (no. 20170540263).

\section{References}

[1] I. Dardalas, E. Stamoula, P. Rigopoulos et al., "Dexmedetomidine effects in different experimental sepsis in vivo models," European Journal of Pharmacology, vol. 856, Article ID 172401, 2019.

[2] X. Chen, Y. Feng, X. Shen et al., "Anti-sepsis protection of Xuebijing injection is mediated by differential regulation of pro- and anti-inflammatory Th17 and T regulatory cells in a murine model of polymicrobial sepsis," Journal of Ethnopharmacology, vol. 211, pp. 358-365, 2018.

[3] H. Shi, Y. Hong, J. Qian, X. Cai, and S. Chen, "Xuebijing in the treatment of patients with sepsis," The American Journal of Emergency Medicine, vol. 35, no. 2, pp. 285-291, 2017.

[4] C. Li, P. Wang, L. Zhang et al., "Efficacy and safety of Xuebijing injection (a Chinese patent) for sepsis: a metaanalysis of randomized controlled trials," Journal of Ethnopharmacology, vol. 224, pp. 512-521, 2018.

[5] W. Wu, R.-1. Jiang, L.-c. Wang et al., "Effect of Shenfu injection on intestinal mucosal barrier in a rat model of sepsis," 
The American Journal of Emergency Medicine, vol. 33, no. 9, pp. 1237-1243, 2015.

[6] Y.-z. Zhang, H.-y. Wu, L.-w. Ren, H.-s. Zhang, X. Jia, and Y.-z. Zhang, "Study on modified ShengMai Yin injection for prevention and treatment of brain impairment in endotoxin shock rats," Journal of Traditional Chinese Medicine, vol. 30, no. 4, pp. 272-277, 2010.

[7] Z. L. Gao, X. Q. Yu, and M. Yang, "Clinical trial of treating stress-induced hyperglycemia patients with sepsis by supplementing Qi, nourishing Yin, and promoting blood flow," Chinese Journal of Integrated Traditional and Western Medicine, vol. 32, no. 10, pp. 1336-1339, 2012.

[8] Y. H. Yu, N. Q. Cui, and G. L. Wang, "Monocyte response and regulatory effect of emodin and shenmai injection on it in patients with severe sepsis," Chinese Journal of Integrated Traditional and Western Medicine, vol. 26, no. Suppl, pp. 98-101, 2006.

[9] Y. H. Yu, N. Q. Cui, Q. Fu, and J. Li, "Change of Th1/Th2 cytokine equilibrium in rats with severe sepsis and therapeutic effect of recombinant interleukin-12 and Shenmai injection," Chinese Journal of Integrative Medicine, vol. 11, no. 2, pp. 136-141, 2005.

[10] S. Zhan, B. Ding, Y.-e. Ruan et al., "A simple blood microdialysis in freely-moving rats for pharmacokinetic-pharmacodynamic modeling study of ShengMai injection with simultaneous determination of drug concentrations and efficacy levels in dialysate," Journal of Pharmaceutical and Biomedical Analysis, vol. 154, pp. 23-30, 2018.

[11] B. Duan, J. Xie, Q. Rui, W. Zhang, and Z. Xi, "Effects of Shengmai injection add-on therapy to chemotherapy in patients with non-small cell lung cancer: a meta-analysis," Supportive Care in Cancer, vol. 26, no. 7, pp. 2103-2111, 2018.

[12] G. H. Yu, W. B. Wang, X. Wang et al., "Network pharmacology-based strategy to investigate pharmacological mechanisms of Zuojinwan for treatment of gastritis," BMC Complementary and Alternative Medicine, vol. 28, p. 292, 2018.

[13] Y. M. Cui, C. H. Li, C. Zeng et al., "Tongmai Yangxin pills anti-oxidative stress alleviates cisplatin-induced cardiotoxicity: network pharmacology analysis and experimental evidence," Biomedicine \& Pharmacotherapy, vol. 108, pp. 2018-1089, 2018.

[14] H. Ming, L. Sha, W. Ning, H. Y. Tan, F. Cheung, and Y. B. Feng, "A biomedical investigation of the hepatoprotective effect of Radix salviae miltiorrhizae and network pharmacology-based prediction of the active compounds and molecular targets," International Journal of Molecular Sciences, vol. 18, no. 3, pp. 620-628, 2017.

[15] Y. Dong, L. Duan, H. W. Chen, Y. M. Liu, Y. Zhang, and J. Wang, "Network pharmacology-based prediction and verification of the targets and mechanism for panax notoginseng saponins against coronary heart disease," EvidenceBased Complementary and Alternative Medicine, vol. 2019, Article ID 6503752, 13 pages, 2019.

[16] F. Tang, Q. Tang, Y. Tian, Q. Fan, Y. Huang, and X. Tan, "Network pharmacology-based prediction of the active ingredients and potential targets of Mahuang Fuzi Xixin decoction for application to allergic rhinitis," Journal of Ethnopharmacology, vol. 176, pp. 402-412, 2015.

[17] C. A. Lipinski, F. Lombardo, B. W. Dominy, and P. J. Feeney, "Experimental and computational approaches to estimate solubility and permeability in drug discovery and development settings," Advanced Drug Delivery Reviews, vol. 46, no. 1-3, pp. 3-26, 2001.
[18] M. Y. Shen, S. Tian, Y. Y. Li et al., "Drug-likeness analysis of traditional Chinese medicines: 1 . property distributions of drug-like compounds, non-drug-like compounds and natural compounds from traditional Chinese medicines," Journal of Cheminformatics, vol. 4, no. 1, p. 31, 2012.

[19] W. Tao, X. Xu, X. Wang et al., "Network pharmacology-based prediction of the active ingredients and potential targets of Chinese herbal radix curcumae formula for application to cardiovascular disease," Journal of Ethnopharmacology, vol. 145, no. 1, pp. 1-10, 2013.

[20] X.-M. Wu and C.-F. Wu, "Network pharmacology: a new approach to unveiling Traditional Chinese Medicine," Chinese Journal of Natural Medicines, vol. 13, no. 1, pp. 1-2, 2015.

[21] S. Ma, C. Feng, X. Zhang et al., "The multi-target capabilities of the compounds in a TCM used to treat sepsis and their in silico pharmacology," Complementary Therapies in Medicine, vol. 21, no. 1, pp. 35-41, 2013.

[22] X. Z. Zhang, W. Xiao, X. J. Xu, Z. Z. Wang, L. Cao, and L. Sun, "Study on mechanism of the reduning injection on the influenza virus using network pharmacology method," Acta Physico-Chimica Sinica, vol. 29, no. 7, pp. 1415-1420, 2013.

[23] Y. Zhang, X. Mao, J. Su et al., "A network pharmacologybased strategy deciphers the underlying molecular mechanisms of Qixuehe capsule in the treatment of menstrual disorders," Chinese Medicine, vol. 12, no. 1, p. 23, 2017.

[24] H. Y. Li, L. H. Zhao, B. Zhang et al., "A network pharmacology approach to determine active compounds and action mechanisms of Ge-Gen-Qin-Lian decoction for treatment of type 2 diabetes," Evidence-Based Complementary and Alternative Medicine, vol. 2014, Article ID 495840, 12 pages, 2014.

[25] A. H. Zhang, "Identifying potential therapeutic targets of a natural product Jujuboside B for insomnia through network pharmacology," Plant Science Today, vol. 1, no. 2, pp. 69-79, 2014.

[26] A. Rhodes, L. E. Evans, W. Alhazzani et al., "Surviving sepsis campaign: international guidelines for management of sepsis and septic shock: 2016," Intensive Care Medicine, vol. 43, no. 3, pp. 304-377, 2017.

[27] P. Valent, E. Hadzijusufovic, G. H. Schernthaner, D. Wolf, D. Rea, and C. Philipp le, "Vascular safety issues in CML patients treated with BCR/ABL1 kinase inhibitors," Blood, vol. 125, no. 6, pp. 901-906, 2014.

[28] A. Quintás-Cardama and J. Cortes, "Molecular biology of bcrabl1-positive chronic myeloid leukemia," Blood, vol. 113, no. 8, pp. 1619-1630, 2009.

[29] Y. Li, H. Wang, K. Tao et al., "MiR-29b suppresses CML cell proliferation and induces apoptosis via regulation of BCR/ ABL1 protein," Experimental Cell Research, vol. 319, no. 8, pp. 1094-1101, 2013.

[30] A. N. Rizzo, J. Aman, G. P. van Nieuw Amerongen, and S. M. Dudek, "Targeting abl kinases to regulate vascular leak during sepsis and acute respiratory distress syndrome," Arteriosclerosis, Thrombosis, and Vascular Biology, vol. 35, no. 5, pp. 1071-1079, 2015.

[31] U. Asghar, A. K. Witkiewicz, N. C. Turner, and E. S. Knudsen, "Targets of the cyclin-dependent kinase Cdk1," Nature, vol. 425, no. 6960, pp. 859-864, 2003.

[32] A. M. Rachael, R. Samuel, C. Elizabeth, L. Thierry, C. Anna, and B. Andrew, "Partial inhibition of Cdk1 in $\mathrm{G}_{2}$ phase overrides the SAC and decouples mitotic events," Cell Cycle, vol. 13, no. 9, pp. 1400-1412, 2014.

[33] Q.-h. Yang, D.-w. Liu, Y. Long, H.-z. Liu, W.-z. Chai, and X.-T. Wang, "Acute renal failure during sepsis: potential role of cell cycle regulation," Journal of Infection, vol. 58, no. 6, pp. 459-464, 2009. 
[34] M. E. Jorrit and D. K. Richard, "An overview of Cdk1-controlled targets and processes," Cell Division, vol. 5, no. 1, p. 11, 2010.

[35] F. Masamitsu, B. G. Steven, W. B. Derry, and H. R. Joel, "Essential embryonic roles of the CKI-1 cyclin-dependent kinase inhibitor in cell-cycle exit and morphogenesis in C elegans," Developmental Biology, vol. 260, no. 1, pp. 273-286, 2003.

[36] R. Honda, H. Tanaka, and H. Yasuda, "Oncoprotein MDM2 is a ubiquitin ligase E3 for tumor suppressor p53," FEBS Letters, vol. 420, no. 1, pp. 25-27, 1997.

[37] Q. Christine, A. S. Todd, and L. Susan, "HSP90 as a capacitor of phenotypic variation," Nature, vol. 417, no. 6889, pp. 618-624, 2002.

[38] F. Michaela-Diana, D. Helen, V. Maria, K. Marianna, I. Stavroula, and B. George, "Increased extracellular heat shock protein $90 \alpha$ in severe sepsis and SIRS associated with multiple organ failure and related to acute inflammatorymetabolic stress response in children," Medicine, vol. 95, no. 35, Article ID e4651, 2016.

[39] A. Conway-Morris, J. Wilson, and M. Shankar-Hari, "Immune activation in sepsis," Critical Care Clinics, vol. 34, no. 1, pp. 29-42, 2017.

[40] H.-Y. Hsu, H.-L. Wu, S.-K. Tan et al., "Geldanamycin interferes with the $90-\mathrm{kDa}$ heat shock protein, affecting lipopolysaccharide-mediated interleukin-1 expression and apoptosis within macrophages," Molecular Pharmacology, vol. 71, no. 1, pp. 344-356, 2007.

[41] A. D. Basso, D. B. Solit, G. Chiosis, B. Giri, P. Tsichlis, and N. Rosen, "Akt forms an intracellular complex with heat shock protein 90 (Hsp90) and Cdc37 and is destabilized by inhibitors of Hsp90 function," Journal of Biological Chemistry, vol. 277, no. 42, pp. 39858-39866, 2002.

[42] M. Broemer, D. Krappmann, and C. Scheidereit, "Requirement of Hsp90 activity for I $\kappa$ B kinase (IKK) biosynthesis and for constitutive and inducible IKK and NF- $\kappa \mathrm{B}$ activation," Oncogene, vol. 23, no. 31, pp. 5378-5386, 2004.

[43] K. Triantafilou, M. Triantafilou, and R. L. Dedrick, "A CD14independent LPS receptor cluster," Nature Immunology, vol. 2, no. 4, pp. 338-345, 2001.

[44] X. P. Li, R. Luo, R. J. Jiang et al., "The role of the Hsp90/Akt pathway in myocardial calpain-induced caspase- 3 activation and apoptosis during sepsis," BMC Cardiovascular Disorders, vol. 13, no. 1, p. 8, 2013.

[45] A. Chatterjee, C. Dimitropoulou, F. Drakopanayiotakis et al., "Heat shock protein 90 inhibitors prolong survival, attenuate inflammation, and reduce lung injury in murine sepsis," American Journal of Respiratory and Critical Care Medicine, vol. 176, no. 7, pp. 667-675, 2007.

[46] E. J. Pone, J. S. Zhang, T. Mai et al., "BCR-signalling synergizes with TLR-signalling for induction of AID and immunoglobulin class-switching through the non-canonical NF- $\kappa \mathrm{B}$ pathway," Nature Communications, vol. 3, no. 1, p. 767, 2012.

[47] J. Huang, K. Liu, S. Zhu et al., "AMPK regulates immunometabolism in sepsis," Brain, Behavior, and Immunity, vol. 72, pp. $89-100,2018$. 